\title{
Le système éducatif dans le sultanat d'Oman
}

\section{Sharifa Al Harthy et Olivier Renard}

\section{OpenEdition}

\section{Journals}

Édition électronique

URL : http://journals.openedition.org/ries/585

DOI : $10.4000 /$ ries. 585

ISSN : 2261-4265

\section{Éditeur}

Centre international d'études pédagogiques

\section{Édition imprimée}

Date de publication : 1 décembre 2008

Pagination : 149-154

ISSN : 1254-4590

\section{Référence électronique}

Sharifa Al Harthy et Olivier Renard, «Le système éducatif dans le sultanat d'Oman », Revue

internationale d'éducation de Sèvres [En ligne], 49 | décembre 2008, mis en ligne le 01 décembre 2011, consulté le 14 novembre 2019. URL : http://journals.openedition.org/ries/585 ; DOI : 10.4000/ries.585

Ce document a été généré automatiquement le 14 novembre 2019.

(c) Tous droits réservés 


\title{
Le système éducatif dans le sultanat d'Oman
}

\author{
Sharifa Al Harthy et Olivier Renard
}

1 Le sultanat d'Oman se situe dans la partie sud-est de la péninsule arabique. Il partage des frontières avec l'Arabie saoudite, les Émirats arabes unis et le Yémen et s'ouvre sur le détroit d'Ormuz, la mer d'Arabie et l'océan Indien. Sa superficie représente environ trois cinquièmes de celle la France, ce qui fait de ce territoire le troisième plus large de la péninsule, pour une population de seulement 2,3 millions de personnes.

2 La structure juridique ainsi que toutes les politiques du sultanat sont issues des directives et décrets du sultan Qaboos bin Said, au pouvoir depuis le 23 juillet 1970, désormais appelé «Jour de la Renaissance ». Le sultan est à la fois chef d'État, premier ministre, commandant en chef des forces armées, ministre de la défense et ministre des affaires étrangères.

3 Sous la direction de cette forte autorité, le gouvernement détermine les orientations, objectifs et politiques du pays. La structure juridique et politique est donc le résultat des orientations choisies par le sultan, elles-mêmes entérinées par le gouvernement.

4 Le sultanat d'Oman a, comme tous ses voisins, des ambitions dans les secteurs touristique, industriel et commercial, mais sa capacité financière est moindre: le produit intérieur brut (PIB) par habitant des Émirats arabes unis ou du Qatar est cinq à six fois plus élevé que celui d'Oman. La pression à la diversification de l'économie omanaise est encore plus forte que dans le reste de la région car les réserves de pétrole exploitables seront probablement épuisées dans à peine plus de dix ans, et le pétrole restant sera difficilement rentable en raison d'une géologie difficile. Dans ces conditions, un choix de long terme semble se dessiner: pour accompagner le développement économique et social engagé depuis son accession au pouvoir, le sultan Qaboos a initié une politique ambitieuse de diffusion du savoir et de financement et de promotion de l'éducation. 


\section{Une volonté politique forte et un développement sans précédent}

5 Dans l'histoire récente d'Oman, c'est-à-dire depuis sa "Renaissance », trois dates ont une signification particulière pour le développement du système éducatif du pays. Dès 1972, lors de son discours annuel, le sultan donne l'impulsion des réformes en expliquant que «l'éducation est [son] grand souci et qu'[il] voit la nécessité de diriger les efforts vers la diffusion de l'éducation. Au ministère de l'éducation ont été donnés l'opportunité et les moyens de briser les chaînes de l'ignorance. Des écoles ont été ouvertes; la chose importante étant qu'il y ait de l'éducation, même à l'ombre des arbres. "

Onze ans plus tard, de nouveau lors de son discours annuel, le sultan renouvelle sa vision et déclare: "la construction de la nation omanaise, la formation de son caractère au travers de l'éducation et de la culture est au premier plan de notre noble cause pour laquelle nous devrons toujours nous battre. »

7 Enfin, en 1995, le sultan ordonne l'organisation d'une conférence pour définir les directions futures du développement économique et social du pays. La vision « Oman 2020 » reconnaît que le monde actuel est dynamique, que l'acquisition des savoirs et des connaissances est essentielle et que le pays doit atteindre les objectifs suivants pour l'année 2020 : un secteur privé efficace et compétitif, une économie ouverte, dynamique et diversifiée, des ressources humaines compétentes et un développement durable dans un environnement stable.

8 Ces résolutions se sont transformées en priorités vers lesquelles tous les participants du système éducatif ont été amenés à diriger leurs efforts. Avant de présenter les stratégies et structures mises en place ainsi que les défis à venir, arrêtons-nous sur quelques images fortes du développement de l'éducation au cours des trente-cinq dernières années. Au début des années 1970, il y avait seulement trois écoles dans tout le sultanat, environ neuf cents écoliers et une trentaine d'enseignants (et aucune université, cela va sans dire). Seulement $35 \%$ de la population savait lire et seulement $12 \%$ parmi les femmes. Aujourd'hui, plus de mille écoles fonctionnent sur l'ensemble du territoire avec plus de 38000 enseignants pour plus de 600000 enfants. Le taux d'illettrés a diminué de $44 \%$ (de $60 \%$ pour les femmes) et aujourd'hui moins de $3 \%$ des jeunes âgés de 15 à 29 ans ne savent pas lire, les garçons et les filles ayant désormais le même accès à l'éducation. Quatre universités ont été ouvertes et les premiers doctorants ont, il y a quelques mois, commencé leurs recherches : à leur naissance, ils vivaient dans un pays au système éducatif quasi-inexistant. Un rapport de la Banque mondiale a décrit le développement du système éducatif omanais comme massif, sans précédent et inégalé par aucun autre pays ${ }^{1}$.

\section{Les plans pour l'éducation préscolaire, primaire et secondaire}

9 La priorité principale du ministère de l'éducation a été jusqu'à récemment de promouvoir l'accès à l'enseignement. Ce n'est que graduellement qu'un recentrage vers la qualité est venu compléter les objectifs quantitatifs. 
10 La politique gouvernementale dans le domaine de l'éducation se fonde sur des plans quinquennaux qui définissent les actions à suivre. Le premier (1976-1980) s'efforça de créer des instituts de formation d'enseignants ainsi que de bâtir des écoles pour remplacer les structures mobiles (tentes, baraques) par des lieux d'éducation aux structures logistiques appropriées. Le deuxième plan (1981-1985) ordonna d'une part la construction de bibliothèques et de salles d'études pour l'enseignement scientifique et les travaux pratiques, et, d'autre part, la transformation des instituts de formation des enseignants en véritables collèges d'enseignement supérieur. Le troisième plan (1986-1990) avait pour objectif de promouvoir l'« omanisation » du corps enseignant, c'est-à-dire le remplacement des enseignants étrangers par des Omanais. Le quatrième plan (1991-1995) continua les efforts précédents et introduisit des éléments de qualité dans les objectifs gouvernementaux. Le cinquième plan (1996-2000) changea fondamentalement le système primaire et engagea des réformes profondes, comme l'introduction de pédagogies centrées sur l'élève en tant qu'individu, la diminution des cours magistraux, l'allongement des durées quotidiennes et annuelles d'enseignement, l'augmentation $\mathrm{du}$ nombre de disciplines et l'introduction de l'enseignement de l'anglais dès le cours préparatoire et de la mixité des classes jusqu'à l'âge de dix ans. Le sixième plan (2001-2005) poursuivit ces directions et s'attacha à offrir à tous l'accès à ce nouveau système. Enfin le septième plan, actuellement en vigueur, prend un virage significatif et indique qu'« il doit y avoir une réorientation de l'accent mis sur l'expansion quantitative de l'offre de services éducatifs vers une amélioration de la qualité du système éducatif.» Cet objectif représente un défi important pour un système qui, malgré ses résultats, reste relativement jeune.

\section{Les défis actuels et les solutions proposées}

11 Même si le ministère de l'éducation se félicite du faible nombre de redoublements à tous les niveaux de l'éducation primaire et secondaire, avec des taux de redoublement de $0,8 \%$ en primaire et de $2 \%$ en secondaire contre des moyennes mondiales de $4,3 \%$ et $3,2 \%$ respectivement, ce prétendu succès cache des résultats qualitatifs décevants. Les études de l'Unesco concluent à la faiblesse du niveau des élèves omanais et une évaluation récente indique une moyenne d'un an de retard par rapport aux standards internationaux et un nombre trois fois plus grand d'élèves ayant des difficultés significatives à lire leur langue maternelle.

12 Les initiatives pour résoudre ces problèmes visent à adopter une approche pragmatique fondée sur un ensemble de politiques de contrôle de la qualité. En particulier, un système d'incitation financière est en train d'être mis en place pour récompenser les succès éventuels des enseignants et de leurs institutions. Parallèlement, le ministère de l'éducation encourage les enseignants à participer à des formations tout au long de leur vie professionnelle. Les besoins de chaque enseignant sont identifiés et des solutions sont proposées afin que les meilleures pratiques pédagogiques soient mises en œuvre dans l'ensemble du sultanat.

13 Ce système de contrôle/incitation/ formation se développe conjointement à un effort de décentralisation visant à transférer les pouvoirs de décision et de gestion des ressources aux établissements eux-mêmes. Ceci s'applique non seulement aux institutions publiques mais aussi, conformément la vision Oman 2020, au secteur privé qui fait l'objet d'une attention particulière. En effet, l'objectif du gouvernement est de 
développer des normes et des cadres visant à encourager le développement de structures innovantes initiées par le secteur privé, qu'il soit omanais ou international. La définition de ces standards aspire à laisser à une variété d'institutions la liberté d'offrir des services éducatifs tout en imposant des contraintes en termes de transparence et de responsabilité. Le succès de cette politique est illustré par l'augmentation du nombre d'écoles privées, de 81 en 1995 à 160 actuellement.

Également importantes sont les initiatives visant à faciliter la transition post-scolaire. Le ministère de l'éducation a coordonné ses efforts avec divers autres ministères et organisations omanaises afin de créer en 2007 un Centre national d'orientation professionnelle qui aide les élèves à choisir leur éducation et leur carrière. De même, les ministères de l'éducation et de l'enseignement supérieur coordonnent actuellement leurs politiques afin de faciliter la transition des élèves du secondaire vers l'enseignement supérieur.

\section{L'enseignement supérieur et la recherche}

15 Seulement $14 \%$ des jeunes Omanais âgés de 18 à 25 ans sont actuellement inscrits à l'université, alors que l'objectif du gouvernement est d'intégrer $50 \%$ de cette classe d'âge dans l'enseignement supérieur. Le premier obstacle à une augmentation massive du nombre d'étudiants dans les cinq à dix ans provient bien sûr des problèmes rencontrés dans l'enseignement primaire et secondaire, qui freinent la transition vers la poursuite des études. Les initiatives décrites précédemment devraient cependant augmenter la demande pour l'enseignement supérieur quand un nombre accru d'élèves aura reçu les fondements requis pour entreprendre des études universitaires. Un second obstacle au développement de l'enseignement supérieur se fera alors certainement sentir: l'offre sera limitée par le nombre insuffisant de professeurs qualifiés.

16 L'unique université publique du pays, Sultan Qaboos University, a ouvert ses portes en 1986 et n'a entrepris d'offrir des programmes de master qu'en 1992. Les premiers programmes doctoraux commencent seulement à être opérationnels. La difficulté est donc claire: le développement de l'enseignement supérieur nécessite un collège important de maîtres de conférence et de professeurs qualifiés. Cela n'est possible qu'à deux conditions : d'une part, des étudiants en nombre suffisant doivent entreprendre dès aujourd'hui des études doctorales pour devenir les professeurs universitaires de demain et, d'autre part, les professeurs actuels doivent avoir les moyens de les encadrer.

17 Cette double condition se transforme en cercle vicieux quand le corps universitaire actuel est débordé par les charges d'enseignement et ne peut s'investir dans la recherche. En outre, la motivation de ces enseignants baisse et leurs connaissances deviennent rapidement obsolètes lorsqu'ils ne sont pas confrontés à des doctorants qui les poussent vers les frontières de la connaissance. En d'autres termes, l'enseignement supérieur omanais éprouvera des difficultés tant que la recherche ne sera pas développée.

18 Pour tenter de remédier à ces problèmes, un Conseil de la recherche a été créé en 2005 sous le patronage du prince Sayid Shihab bin Tareq Al Saeed. Les objectifs assignés à cette instance comprennent la mise en place d'une stratégie planifiée pour la recherche scientifique, la préparation d'un programme de mise en œuvre visant à encourager le 
développement de la recherche et la créativité individuelle dans le cadre de priorités définies au niveau national, ainsi que la mise en place de mécanismes de diffusion du savoir. Un cadre organisationnel a été défini après une série de consultations et de travaux dirigés par la communauté académique et des représentants des secteurs public et privé. Cette organisation est amenée à se développer dans les prochaines années en définissant ses propres régulations afin de créer une agence de moyens qui réponde efficacement aux besoins du sultanat.

19 Par sa culture du débat, l'activité de recherche soumet toute hypothèse à la critique, c'est-à-dire à la réfutation permanente, ce qui réduit le champ des idéologies : tout résultat rationnel, aussi établi qu'il paraisse, reste soumis à l'évaluation et appelle des corrections ultérieures. Tout comme l'éducation dont elle est la suite logique, la recherche académique forme ainsi les esprits et augmente sensiblement un capital humain qui est au centre même des objectifs de politiques visant à trouver des alternatives au pétrole. Dans une société fondée sur la connaissance, l'effort intellectuel demandé par la méthodologie de la recherche est un excellent entraînement à la formation de l'esprit critique.

Ceci étant, pour qu'un système de recherche et d'enseignement supérieur fonctionne, il faut un nombre suffisamment important d'opérateurs de recherche. Or les chiffres ne jouent pas en faveur d'Oman : il y a plus de chercheurs en sciences du végétal et de l'animal en Aquitaine que dans tout le sultanat, tous domaines confondus. La réponse à cet obstacle est double: augmenter les ressources humaines et les focaliser sur un nombre limité de projets. Tels sont les véritables éléments de la stratégie de l'enseignement supérieur et de la recherche pour Oman, déterminant ainsi les mécanismes de financement sur projets - ce qui fera sans doute écho à des problématiques françaises. Le choix de cette option stratégique dans le développement de l'enseignent supérieur a été discuté tout au long de l'année 2007 par une multitude de parties, incluant différents ministres et présidents d'universités.

Il s'agissait du choix entre, d'une part, un modèle centré sur une agence de moyens comme l'Agence nationale de la recherche (ANR) en France qui sélectionne des projets retenus dans le cadre d'appels à projets après mise en concurrence et évaluation par les pairs et, d'autre part, un modèle dans lequel des opérateurs de recherche sont autonomes dans l'utilisation de financements de base, c'est-à-dire à plus long terme et affectés à des équipes plutôt qu'à des projets précis. Ces opérateurs de recherche (par exemple le CEA, le CNRS - en tout cas jusqu'à aujourd'hui, l'INSERM, et l'INRA en France) ont pour caractéristique de définir eux-mêmes leur stratégie selon une procédure collégiale.

Quels sont donc les paramètres qui ont amené Oman à faire le choix d'une coordination plus centralisée et prescriptive? Une tradition dans laquelle la population se tourne généralement vers un État fort n'explique pas tout. Le gouvernement omanais a en effet mis en place dans beaucoup de domaines, y compris dans l'éducation comme on vient de le voir, des processus d'autonomisation et de décentralisation des décisions. La structure politique du sultanat ne suffit donc pas à comprendre ce choix. Plutôt, comme nous l'avons souligné, le nombre d'enseignants-chercheurs à l'université omanaise est très réduit et chaque chercheur peine à trouver des collègues qui vont s'orienter vers des thèmes de recherche contigus; bien souvent, ils sont amenés à ne pouvoir collaborer qu'avec leurs anciens directeurs de thèse à l'étranger, sur des thèmes qui sont fréquemment bien éloignés des problématiques locales. Là où la 
France, avec plus de 75000 chercheurs et enseignants-chercheurs dans le secteur public, devrait laisser à ses acteurs de recherche la liberté de définir leur portefeuille de projets - et respecter ainsi la puissance créatrice née d'une masse critique de chercheurs indépendants - Oman se doit d'être plus directif et de faire en sorte que les acteurs puissent se coordonner sur quelques sujets afin de ne pas s'éparpiller. Même s'il se peut qu'une telle intrusion dans la liberté académique ait un coût, Oman, étant au début du développement de son système de recherche, a besoin de directions stratégiques plus étroites, tout en gardant à l'esprit qu'il faudra desserrer l'étau quand le problème de masse critique commencera à être moins aigu.

\section{Un appel aux chercheurs en politiques de l'éducation}

Le sultanat d'Oman est un immense laboratoire pour l'étude des systèmes éducatifs. Voici à peine trente-cinq ans, les enfants recevaient leur éducation du chef religieux ou du sage local, souvent à l'ombre d'un arbre. L'argent du pétrole et la vision du sultan ont transformé le paysage éducatif omanais. Les garçons et les filles d'Oman peuvent recevoir une éducation qui va de la maternelle à l'université. Aucun autre pays n'a transformé son système éducatif de la sorte et cela seul mériterait une attention plus approfondie que ce présent éclairage ne peut fournir.

Plus encore, l'avenir présente des défis immenses quant aux prochaines transformations nécessaires à l'absorption de masses significatives de jeunes en demande de formation. La fin programmée du pétrole à Oman, qui pourrait frapper le pays dans les quinze ou vingt prochaines années, va susciter une exigence de connaissances et de savoirs pour diversifier l'économie. Le système éducatif, qui a su se développer, va donc devoir accélérer sa transformation pour offrir une éducation appropriée. Afin que les hommes politiques et les décideurs omanais fassent les bons choix de structure, de gouvernance, de curriculum et de financement, des recherches académiques sont indispensables.

Quelques données sur le sultanat d'Oman

\begin{tabular}{|l|l|}
\hline Superficie & $309500 \mathrm{~km}^{2}$ \\
\hline $\begin{array}{l}\text { Population } \\
\text { Omanais } \\
\text { Expatriés }\end{array}$ & $\begin{array}{l}1781360 \\
559455\end{array}$ \\
\hline Densité & $7,6 \mathrm{hab} / \mathrm{km}^{2}$ \\
\hline $\begin{array}{l}\text { Espérance de vie } \\
\text { hommes } \\
\text { femmes }\end{array}$ & $72,2 \mathrm{ans}$ \\
\hline Nombre moyen d'enfants par famille & 5,3 \\
\hline PIB par habitant & $75,4 \mathrm{ans}$ \\
\hline Croissance annuelle du PIB & $58 \%$ \\
\hline
\end{tabular}




\begin{tabular}{|l|l|}
\hline Part des produits pétroliers & $25 \%$ \\
dans le PIB & $84 \%$ \\
dans les exportations & \\
\hline Taux de scolarisation & $7 \%$ \\
(par rapport à la classe d'âge) & $83 \%$ \\
Maternelle & $63 \%$ \\
Primaire & $14 \%$ \\
Secondaire & \\
Supérieur & \\
\hline
\end{tabular}

Données pour 2006

\section{NOTES}

1. L'espérance de vie est de 72,2 ans pour les hommes et de 75,4 pour les femmes, alors qu'elle était de 40 ans en 1970.

\section{INDEX}

Mots-clés : enseignement primaire, enseignement secondaire, enseignement supérieur, évaluation, recherche, système éducatif

Index géographique : Oman

\section{AUTEURS}

\section{SHARIFA AL HARTHY}

Assistante de recherche, Conseil de la recherche du sultanat d'Oman

\section{OLIVIER RENARD}

Conseiller spécial du secrétaire général du Conseil de la recherche du sultanat d'Oman 